Introduction The pressure for faster, cheaper, better sample testing has resulted in an increasing need for multiplexed assays. Current real-time chemistries are expensive and/or difficult to multiplex. MNAzyme qPCR is a novel real-time technology that provides a powerful tool for molecular diagnostics with multiple advantages over other real-time chemistries. A multiplex MNAzyme qPCR panel was used for the identification of causative bacterial pathogens important for prompt and proper treatment of STIs.

Methods An MNAzyme qPCR panel was developed for the multiplexed detection of Mycoplasma genitalium, Mycoplasma hominis, Ureaplasma parvum and Ureaplasma urealyticum together with a DNA extraction control. MNAzymes are comprised of two DNA partzymes, which come together only in the presence of PCR amplicons, to form active enzymes that modify universal reporter probes and produce signals that are monitored in realtime. Since signal production requires the binding of two partzymes and two PCR primers to a target, this approach has higher specificity than other chemistries, making it ideal for molecular diagnostics. The use of a series of well-characterised, universal probes provides many advantages over target specific probes. They result in reliable, consistent performance when coupled to any target and facilitate rapid and simple development of multiplex tests.

Results The STI panel showed robust performance in multiplex with high analytical specificity and sensitivity. The $\mathrm{R}^{2}$ for all targets was above 0.99 and PCR efficiencies ranged from $90 \%$ to $110 \%$. No inter-panel cross-reactivity was observed and no cross-reactivity was detected using a wide range of non-target organisms.

Conclusion MNAzyme qPCR provides a superior approach to real-time PCR with the advantages of greater specificity, robust multiplex performance, reduced cost, and it is more amenable than other technologies for the rapid development of multiplex assays. These features have been demonstrated through the STI MNAzyme qPCR panel.

Disclosure of interest statement SpeeDx is the developer and manufacturer of the assay evaluated in this study.

\section{P07.08 DESIGNING OF MOLECULAR BEACON BASED POLYMERASE CHAIN REACTION METHOD AS AN UNCONVENTIONAL LOW COST DIAGNOSTIC ASSAY FOR SEXUALLY TRANSMITTED DISEASES}

D Sachdev, SC Sonkar, AL Patel, D Saluja*. Dr B R Ambedkar Center for Biomedical Research, University of Delhi, Delhi-110007, India

\subsection{6/sextrans-2015-052270.324}

Introduction About 300 million new infections of gonorrhoea, Chlamydia, or trichomoniasis occur each year. Young adults, population with health inequities and people in resource poor settings bear the significant proportion of sexually transmitted infections (STI). Therefore, rapid, inexpensive and acceptable tests are needed to address STIs in developing countries. PCR based tests are recommended for diagnosis of several STIs, but such tests require sophisticated infrastructure, need to be imported and hence cannot be used in resource limited settings. Affordability can be countered by the development of in house diagnostic assays which are practical and easy to use, and produced within country to further lower the cost.
Methods Endocervical swabs were collected from patients visiting gynaecology department of hospitals in Delhi. In-house PCR based assay was developed for C. trachomatis, N. gonorrhoeae and $T$. vaginalis and modified to visual assay using molecular beacon for end-point detection.

Results The molecular beacon based PCR assay was developed for C. trachomatis, N. gonorrhoeae and T. vaginalis and evaluated against different commercial kits viz; Roche AMPLICOR NG/CT kit and qPCR kit of Abbott and fast-track diagnostics. Specificity of molecular beacon was confirmed by competition experiments. Diagnostic tests were more than $95 \%$ specific and 99.0\% sensitive for all the three pathogens and negative and positive predicted values were around $98.5 \%$ and $97.5 \%$, respectively at $95 \%$ CI for these three pathogens. We also observed that dry swab samples gave concordant results with that of wet swabs. Assay reagents were stable for more than 6 months at room temperature. We also report, high rates of co-infection for C. trachomatis + N. gonorrhoeae, C. trachomatis + T. vaginalis, N. gonorrhoeae + T. vaginalis amongst women patients in India. Conclusions Development of a rapid, sensitive, specific and PCR based visual diagnostic assay, suitable for developing countries will provide a better disease management.

\section{P07.09 A VALIDATION STUDY OF THE CEPHEID XPERTÂ CT/NG FOR DETECTING CHLAMYDIA TRACHOMATIS AND NEISSERIA GONORRHOEAE IN RECTAL SAMPLES}

${ }^{1}$ LA Cosentino*, ${ }^{2} \mathrm{CS}$ Danby, 'LK Rabe, ${ }^{1} \mathrm{CL}$ Priest, ${ }^{1} \mathrm{KC}$ Damare, 'IS Macio, 'LA Meyn, ${ }^{1,2} \mathrm{HC}$ Wiesenfeld, 1,2 $\mathrm{SL}$ Hillier. 'Magee-Womens Research Institute; ${ }^{2}$ The University of Pittsburgh, Department of Obstetrics, Gynecology and Reproductive Sciences, Pittsburgh, $P A, U S A$

\subsection{6/sextrans-2015-052270.325}

Introduction The objective of this study was to compare the Cepheid Xpert CT/NG (Xpert) and Gen-Probe Aptima (AC2) systems for the identification of Chlamydia trachomatis (CT) and Neisseria gonorrhoeae (GC) from rectal swabs.

Methods Anorectal swabs were collected from 399 participants (224 men and 175 women) who reported a lifetime history of anal receptive intercourse. True positives for this study were defined if both AC2 and Xpert were positive, or if either AC2 or Xpert were positive and the APTIMA CT or APTIMA GC, which target alternate primers, were positive.

Results There was a high level of agreement between Xpert and AC2 for detection of CT and GC from rectal swabs, with only 2/30 discrepant results for GC and 9/59 discrepant results for CT. Following resolution of discrepants, rectal swabs were positive for CT in $58(14.5 \%)$ and for GC in $30(7.5 \%)$ of the 399 participants. For CT, the sensitivity and specificity of Xpert (96.6\%, 99.7\%) and AC2 (91.4\%, 100\%) were comparable, with men and women both having high frequencies of rectal CT $(17.4 \%$ vs $10.9 \%, \mathrm{P}=0.08)$. For $\mathrm{GC}$, the sensitivity and specificity of Xpert $(100 \%, 100 \%)$ and AC2 $(93.3 \%, 100 \%)$ were similar. However, rectal GC was detected much more frequently in men than in women $(11.6 \%$ vs $2.3 \%, \mathrm{P}<0.001)$.

Conclusion Rectal GC and CT were common among people reporting a history of receptive anal sex. Xpert and AC2 had high levels of agreement for rectal swab samples.

Disclosure of interest statement Reagents for CT/GC testing were provided for by Cepheid and Hologic. 